\title{
Distributed spectrum leasing via vertical cooperation in spectrum sharing networks
}

\author{
Yuzhe Xu, Liping Wang, Carlo Fischione and Viktoria Fodor \\ School of Electrical Engineering and the ACCESS Linnaeus Center \\ KTH Royal Institute of Technology \\ Email: \{yuzhe, lipingw, carlofi, vfodor\}@kth.se
}

\begin{abstract}
In this paper, a hierarchical cognitive radio network is considered, where unlicensed secondary users can maximize the transmission capacity by cooperating with licensed primary users. An asynchronous cognitive radio network and average interference model is assumed. An maximization of the secondary users capacity is proposed by controlling the transmit radio power, the secondary users relaying selection, and power splitting while guaranteeing primary users performance. A method is developed to find the solution to such a challenging mixed integer and non convex problem. The method provides an algorithm for finding the optimal power allocations for secondary users, and a greedy algorithm to finding sub-optimal associations between primary and secondary users. Numerical results evaluate the performance of the proposed method.
\end{abstract}

\section{INTRODUCTION}

We consider hierarchical cognitive radio networks (CRNs), where licensed primary users (PUs) and unlicensed secondary users (SUs) coexist in the same region and share the same spectrum. The primary users hold performance guarantees (or have priority to access the spectrum), whereas the secondary users need to be cognitive towards the activities of the PUs and access the spectrum conservatively to conform the constraints from the PUs.

In the spectrum leasing model (or the property rights model) of CRNs, primary users possibly lease part of the owned radio resource to secondary users in exchange for some form of compensation. An efficient way to lease spectrum is based on vertical cooperation, where the SUs can help the primaries to maintain their QoS by cooperatively relaying primary traffic, and simultaneously gain transmission opportunity for themselves as a reward.

In [1], [2], [3], [4] the primary link can lease the owned bandwidth for a fraction of time to a subset of secondary transmitters in exchange of cooperative SU relaying in the form of transmission via distributed space-time coding (DSTC). SU relay selection and time allocation of such time-based spectrum leasing system have been studied by using the methods of optimization [1], [4] and game theory [2], [3].

We consider power-based spectrum leasing [5], [6], where each transmission time slot is divided into two time phases, if a selected SU transmitter can successfully decode the primary signal transmitted in the first time fraction, it regenerates and linearly combined with the secondary signal by assigning fraction $\alpha$ and $(1-\alpha)$ of the available power to the primary and secondary signals respectively, and than broadcasts the combined signal in the secondary transmission phase.

Although there is substantial research related to resource allocation or scheduling for spectrum leasing CRNs, most of the results focus on the power allocation in the physical layer, and moreover, consider the simplified scenario with only one primary link in the network. In this paper, we consider multiple concurrent primary transmissions in the network, and we aim to perform cross-layer optimization including optimal relay selection, scheduling, power allocation, and splitting.

Distributed cross-layer algorithms for standalone ad hoc network have been designed in [7] [8]. Compared with their works, our problem for spectrum sharing networks is substantially more complicated because we consider the interference between the two coexisting networks (the primary and the secondary), and we guarantee the performance of the primary network while maximizing the performance of the secondaries.

\section{System Model}

We consider a cognitive radio network with the following characteristics:

- Multiple primary transmitter and receiver pairs are randomly located in the network;

- Multiple secondary transmitter and receiver pairs coexist with the primary ones and are allowed to access the spectrum via vertical cooperation if the constraints from the primaries are obeyed;

- The vertical cooperation scheme we took into consideration is described as follows: for a primary transmitter and receiver pair (PT-PR));

- If no secondary pair is selected, the PT transmits signal directly to the PR as Fig. 1(a);

- With a secondary pair ST-SR (Secondary User Transmitter-Secondary User Receiver) selected, a time slot is divided into two phases. In the first transmission phase, the primary transmitter (PT) transmits the primary signal to a primary user receiver (PR), which is also received and then decoded by ST and SR. At ST, the primary signal is regenerated and linearly combined with the secondary signal by assigning fractions $\alpha$ and $(1-\alpha)$ of the available power to the primary and secondary signals, respectively. This combined signal is then broadcasted by the ST in the second transmission phase. 


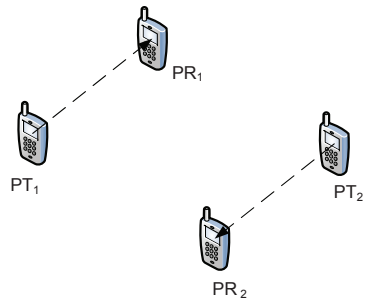

(a)

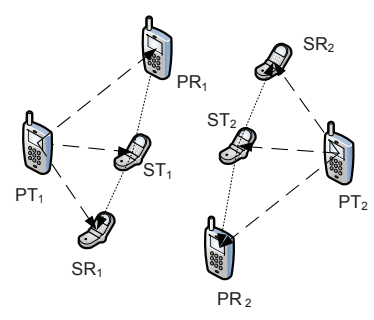

(b)
Fig. 1. Example of (a) a standalone primary network with spectrum reuse, and (b) coexisting primary and secondary network with spectrum leasing.
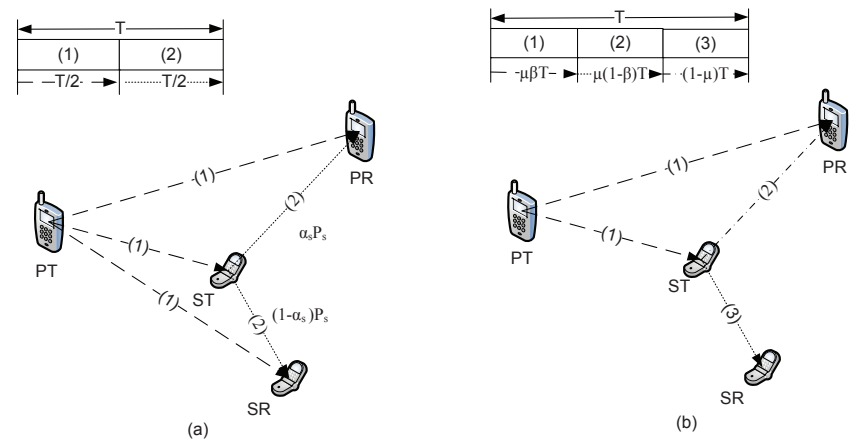

Fig. 2. Two representative spectrum leasing method (a) power splitting relaying, and (b) time-division relaying. We consider the power splitting relaying in this work.

The network model and the spectrum leasing scheme are illustrated in Fig. 1(b) and 2(a), respectively.

\section{Problem Formulation}

Consider $N_{\mathrm{P}}$ primary transmitter and receiver pairs randomly located in a cognitive radio network. Let $\mathcal{P}$ denote the set of the primary pairs. The transmission power of primary users is fixed and given by vector $\tilde{\mathbf{Q}}_{\mathrm{P}}=\left(\tilde{Q}_{1}, \ldots, \tilde{Q}_{N_{\mathrm{P}}}\right)^{T}$. The link capacity for a standalone primary network with direct transmission is denoted by vector $\tilde{\mathbf{C}}_{\mathrm{P}}=\left(\tilde{C}_{1}, \ldots, \tilde{C}_{N_{\mathrm{P}}}\right)^{T}$. The target or the performance requirement of the primary pairs is defined as vector $\hat{\mathbf{C}}_{\mathrm{P}}$. Moreover, the primary users should operate with a certain margin $\rho<1$, i.e., $\hat{\mathbf{C}}_{\mathrm{P}}=\rho \tilde{\mathbf{C}}_{\mathrm{P}}$, which allows to accommodate transmissions in the secondary system [9].

Consider $N_{\mathrm{S}}$ randomly located secondary transmitterreceiver pairs coexist with the primary pairs in the cognitive radio network. Let $\mathcal{S}$ denote the secondary pairs. The transmission power of secondary users is denoted by vector $\mathbf{P}_{\mathrm{S}}=\left(P_{1}, \ldots, P_{N_{\mathrm{S}}}\right)^{T}$, and the power splitting is denoted as vector $\boldsymbol{\alpha}=\left(\alpha_{1}, \ldots, \alpha_{N_{\mathrm{S}}}\right)^{T}$. Moreover, the matrix $\boldsymbol{\beta} \in \mathbb{R}^{N_{\mathrm{P}} \times N_{\mathrm{S}}}$ indicates the association between primary and secondary users. Namely, the entry $[\boldsymbol{\beta}]_{i j}=1$ if $i$-th primary pair selects $j$ th secondary pair as relay, otherwise it is 0 . To avoid heavy notation, we let $\beta=\left\{\beta_{p}^{s}, \forall p \in \mathcal{P}, s \in \mathcal{S}\right\}$. Note that in this paper, we want to find the optimal cooperation strategy that is specified by the optimal $\mathbf{P}_{\mathrm{S}}^{*}, \boldsymbol{\alpha}^{*}$ and $\boldsymbol{\beta}^{*}$.

However, consider the link capacity and interference model, which will be formally described later, find the optimal solu- tions $\mathbf{P}_{\mathrm{S}}^{*}, \boldsymbol{\alpha}^{*}$ and $\boldsymbol{\beta}^{*}$ are Mixed-Integer and non-convex problem (MINCop) that in general is NP-hard. In this paper, we divide this MINCop problem into two related subproblems. One is the secondary user power allocation problem providing optimal solutions for $\mathbf{P}_{\mathrm{S}}$ and $\boldsymbol{\alpha}$ with respect to a fixed $\boldsymbol{\beta}$. The other is the secondary user selection problem that aims to provide optimal solution for $\boldsymbol{\beta}$. Note that the secondary user selection problem is still an NP hard problem in general.

Following optimization problem represents the entire problem with variables $\mathbf{P}_{\mathrm{S}}, \boldsymbol{\alpha}$ and $\boldsymbol{\beta}$ :

$$
\begin{aligned}
\max _{\mathbf{P}_{\mathrm{S}}, \boldsymbol{\alpha}, \boldsymbol{\beta}} & \min _{s \in \mathcal{S}} C_{s} \\
\text { s.t. } & \overline{\mathbf{C}}_{\mathrm{P}} \geq \hat{\mathbf{C}}_{\mathrm{P}} \\
& \beta_{p}^{s} \in\{0,1\}, \forall p, s \\
& \sum_{s \in \mathcal{S}} \beta_{p}^{s} \leq 1, \forall p \\
& \sum_{p \in \mathcal{P}} \beta_{p}^{s} \leq 1, \forall s \\
& 0 \leq P_{s} \leq P_{s}^{\max }, \forall s \\
& 0 \leq \alpha_{s} \leq 1, \forall s .
\end{aligned}
$$

Considering fairness and throughput, the aim of problem (1) is to maximize the minimum capacity of secondary users guaranteeing the target performance of the primal pairs, by Eq. (1b). The variables are binary number $\beta_{p}^{s}$, real number $\alpha_{s}$ and $P_{s}$. Here $\beta_{p}^{s}=1$ if and only if primary pair $p$ uses secondary pair $s$ as relay. Moreover, every primal pair needs at most one relay, while every secondary pair can serve as one relay, which is formally described by Eq. (1d) and (1e). Eq. (1f) and (1g) indicates the constraints on the transmission power and power splitting for secondary users.

Moreover, for primary users, the link capacity is defined as following:

$$
\bar{C}_{p}=\left(1-\sum_{s} \beta_{p}^{s}\right) C_{p}^{\mathrm{dir}}+\sum_{s} \beta_{p}^{s} C_{p, s}^{\mathrm{cop}},
$$

where

$$
\begin{gathered}
C_{p}^{\mathrm{dir}}=\log \left(1+\frac{Q_{p} G_{p p^{\prime}}}{\sigma^{2}+I_{p p^{\prime}}}\right), \\
C_{p, s}^{\mathrm{cop}}=\frac{1}{2} \min \left\{C_{p 2 s}, C_{p 2 s^{\prime}}, C_{p s 2 p^{\prime}}\right\},
\end{gathered}
$$

with

$$
\begin{gathered}
C_{p 2 s}=\log \left(1+\frac{Q_{p} G_{p s}}{\sigma^{2}+I_{p s}}\right), \\
C_{p 2 s^{\prime}}=\log \left(1+\frac{Q_{p} G_{p s^{\prime}}}{\sigma^{2}+I_{p s^{\prime}}}\right), \\
C_{p s 2 p^{\prime}}=\log \left(1+\frac{Q_{p} G_{p p^{\prime}}}{\sigma^{2}+I_{p p^{\prime}}}+\frac{\alpha_{s} P_{s} G_{s p^{\prime}}}{\sigma^{2}+I_{s p^{\prime}}+\left(1-\alpha_{s}\right) P_{s} G_{s p^{\prime}}}\right),
\end{gathered}
$$

where $\sigma^{2}$ is the average noise power in the network, and $I_{i j}$ denotes interference with for transmitter $i$ and receiver $j$. Moreover, superscript 'dir' and 'cop' indicates direct transition 
and cooperation transition respectively. Note that for primal pair $p$, we use $p, p^{\prime}$ denote the transmitter, receiver respectively. Similarly, $s, s^{\prime}$ are the transmitter, receiver in secondary pair respectively. For secondary users, we have

$$
C_{s}=\left(1-\sum_{p} \beta_{p}^{s}\right) C_{s}^{\mathrm{dir}}+\sum_{p} \beta_{p}^{s} C_{s, p}^{\mathrm{cop}},
$$

where

$$
\begin{gathered}
C_{s}^{\mathrm{dir}}=\log \left(1+\frac{P_{s} G_{s s^{\prime}}}{\sigma^{2}+I_{s s^{\prime}}}\right), \\
C_{s, p}^{\mathrm{cop}}=\frac{1}{2} \log \left(1+\frac{\left(1-\alpha_{s}\right) P_{s} G_{s s^{\prime}}}{\sigma^{2}+I_{s s^{\prime}}}\right) .
\end{gathered}
$$

We consider asynchronous system and use the average interference model:

$$
\begin{aligned}
I_{p p^{\prime}}= & \sum_{w \in \mathcal{P}_{-p}}\left\{\left(1-\sum_{s} \beta_{w}^{s}\right) Q_{w} G_{w p^{\prime}}+\frac{1}{2} \sum_{s} \beta_{w}^{s}\left(Q_{w} G_{w p^{\prime}}\right.\right. \\
& \left.\left.+P_{s} G_{s p^{\prime}}\right)\right\}+\sum_{s}\left(1-\sum_{w \in \mathcal{P}-p} \beta_{w}^{s}\right) P_{s} G_{s p^{\prime}},
\end{aligned}
$$

while $I_{p s}, I_{p s^{\prime}}, I_{s p}$ and $I_{s s^{\prime}}$ can be given with similar expressions that are omitted here.

\section{Solution AND Algorithm}

To derive the solutions to problem (1), we consider the following equivalent problem:

$$
\begin{array}{rl}
\max _{\boldsymbol{\beta}, \boldsymbol{\alpha}, \mathbf{P}_{s}, t} & t \\
\text { s.t. } & C_{s} \geq t, \forall s, \\
& \text { Constraints }(1 \mathrm{~b})-(1 \mathrm{~g})
\end{array}
$$

The procedure for solving the problem is established by two phases: power allocation and secondary user selection. First, we solve the power allocation problem to find optimal $\mathbf{P}$ and $\boldsymbol{\alpha}$ assuming that the solution to selection problem, $\boldsymbol{\beta}$, is given. In secondary user selection problem, we find the optimal solutions by using the results of the power allocation problem. Before giving the detail of the solution procedure, we introduce following assumption for the centralized solution.

Assumption 1. Suppose there exists a central controller. The knowledge of antenna gains, i.e., $G_{i j}$ for all transmitter $i$ and receiver $j$, are known by this controller. Moreover, the central controller distributes the value of $t$ and the association matrix $\boldsymbol{\beta}$ in the network.

Note that under the previous assumption, the central controller decides the capacity targets for secondary users, and assign secondary users to primal users for better performance.

\section{A. Power Allocation Problem}

Assuming $\boldsymbol{\beta}$ is given, problem (12) has following Lemma which gives Pareto-optimal boundary.

Lemma 1. Let $\sigma^{2}>0$ be any positive value, and let $\mathbf{P}_{S}^{*}$ and $\boldsymbol{\alpha}^{*}$ be the optimal solutions to problem (1) given fixed $\boldsymbol{\beta}$. Then, there exists secondary user $s$ such that $P_{s}^{*}=P_{s}^{\max }$ or exists primal user $p$ such that $C_{\mathrm{p}}\left(\mathbf{P}_{S}^{*}, \boldsymbol{\alpha}^{*}\right)=\hat{C}_{\mathrm{p}}$.

Proof: Suppose that in the optimal solution, all secondary users have $\mathbf{P}_{\mathrm{S}}^{*}<\mathbf{P}_{\mathrm{S}}^{\max }$ and all primal users have $\mathbf{C}_{\mathrm{p}}\left(\mathbf{P}_{\mathrm{S}}^{*}, \boldsymbol{\alpha}^{*}\right)>$ $\hat{\mathbf{C}}_{\mathrm{p}}$. Then, there exists scalar $a>1$, such that $a \mathbf{P}_{\mathrm{S}}^{*} \leq \mathbf{P}_{\mathrm{S}}^{\max }$ and $\mathbf{C}_{\mathrm{p}}\left(a \mathbf{P}_{\mathrm{S}}^{*}, \boldsymbol{\alpha}^{*}\right) \geq \hat{\mathbf{C}}_{\mathrm{p}}$. Note that the capacities of secondary users fulfill $C\left(a \mathbf{P}_{\mathrm{S}}\right) \geq C\left(\mathbf{P}_{\mathrm{S}}\right)$ for all $a>1$. Thus $a \mathbf{P}_{\mathrm{S}}^{*}$ is not only a feasible solution but also has better capacities for secondary users. It contradicts to the assumption $\mathbf{P}_{\mathrm{S}}^{*}$ is optimal, which completes the proof.

Moreover, fix secondary selection $\boldsymbol{\beta}$, and consider following power allocation problem that aims to minimize the sum of transmission power:

$$
\begin{array}{ll}
\min _{\boldsymbol{\alpha}, \mathbf{P}_{\mathrm{S}}} & \sum_{s} P_{s} \\
\text { s.t. } & \overline{\mathbf{C}}_{\mathrm{P}} \geq \hat{\mathbf{C}}_{\mathrm{P}} \\
& C_{s} \geq t, \forall s \\
& 0 \leq P_{s} \leq P_{s}^{\max }, \forall s \\
& 0 \leq \alpha_{s} \leq 1, \forall s,
\end{array}
$$

in which $t$ is given. Note that if the optimal solution $t^{*}$ for problem (12) is known, then the optimal solutions $\boldsymbol{\alpha}, \mathbf{P}_{\mathrm{S}}$ for it can be determined by solving problem (13). Formally, we conclude this observation as following lemma.

Lemma 2. Let $\boldsymbol{\beta}$ fixed. Then, the maximum minimum link capacity for secondary users $t^{\prime}$ is feasible for problem (12) if and only if it is feasible for problem (13).

Proof: The proof is straightforward, and is omitted here.

The previous lemma indicates that the largest feasible $t$ for problem (13) is optimal solution $t^{*}$ for problem (12). Before turning to the algorithm, we need following proposition. Then we have following proposition:

Proposition 3. Given $t$, consider following optimization problem:

$$
\begin{array}{ll}
\min _{\boldsymbol{\alpha}, \mathbf{P}_{s}} & \sum_{s} P_{s} \\
\text { s.t. } & P_{s}^{\mathrm{dir}} \geq \frac{2^{t}-1}{G_{s s^{\prime}}}\left(\sigma^{2}+I_{s s^{\prime}}\right) \\
& P_{s}^{\mathrm{cop}} \geq \frac{2^{2 t}-1}{\left(1-\alpha_{s}\right) G_{s s^{\prime}}}\left(\sigma^{2}+I_{s s^{\prime}}\right) \\
& P_{s}^{\mathrm{cop}} \geq \frac{\sigma^{2}+I_{s p^{\prime}}}{G_{s p^{\prime}}\left(\alpha_{s} / A-1+\alpha_{s}\right)} \\
& 0 \leq P_{s} \leq P_{s}^{\max }, \forall s \\
& 0 \leq \alpha_{s} \leq 1, \forall s,
\end{array}
$$

where $A$ is short for $2^{2 \hat{C}_{P}}-1-Q_{P} G_{p p^{\prime}} /\left(\sigma^{2}+I_{p p^{\prime}}\right)$. If optimal solutions for problem (14) are feasible for problem (13), then they are the optimal solutions for problem (13). Otherwise, problem (13) is infeasible given $t$.

Before proving this Proposition, we need following definition and lemma: Refer to [10], recall the definition of standard interference function: 
Definition 1. A function $\mathbf{I}(\mathbf{p})$ is standard interference function if for all $\mathbf{p} \geq 0$ the following properties are satisfied.

- Positivity: $\mathbf{I}(\mathbf{p})>0$ for all $\mathbf{p}$

- Monotonicity: If $\mathbf{p} \geq \mathbf{p}^{\prime}$, then $\mathbf{I}(\mathbf{p}) \geq \mathbf{I}\left(\mathbf{p}^{\prime}\right)$

- Scalability: For all scalar $\alpha>1, \alpha \mathbf{I}(\mathbf{p})>\mathbf{I}(\alpha \mathbf{p})$

Note that the interference introduced in Problem (1) is standard interference function.

Lemma 4. If problem (14) is feasible for some $\mathbf{P}_{S}$, then there exists an unique optimal power allocation $\mathbf{P}_{S}^{*}$ such that constraint (14b), (14c) and (14d) hold at equality.

Proof: Suppose that problem (14) is feasible, and let $\boldsymbol{\alpha}^{*}$ and $\mathbf{P}_{\mathrm{S}}^{*}$ be the optimal power allocation. Denote the right side of constraint (14b), (14c) and (14d) by $\mathbf{I}_{1}\left(\mathbf{P}_{\mathrm{S}}\right), \mathbf{I}_{2}\left(\mathbf{P}_{\mathrm{S}}, \boldsymbol{\alpha}\right)$, $\mathbf{I}_{3}\left(\mathbf{P}_{\mathrm{S}}, \boldsymbol{\alpha}\right)$ respectively. Note that $\mathbf{I}_{i}$ for all $i \in\{1,2,3\}$ are standard interference function in $\mathbf{P}_{\mathrm{S}}$, while $\mathbf{I}_{2}$ and $\mathbf{I}_{3}$ are monotonously increasing and decreasing respectively in $\boldsymbol{\alpha}$. Thus we need to prove that $\mathbf{P}_{\mathrm{S}}^{*}=\mathbf{I}_{i}\left(\mathbf{P}_{\mathrm{S}}^{*}, \boldsymbol{\alpha}^{*}\right)$ for all $i \in\{1,2,3\}$.

Suppose there exists secondary user $s$ such that $\left[\mathbf{P}_{\mathrm{S}}^{*}\right]_{s}>$ $\left[\mathbf{I}_{1}\left(\mathbf{P}_{\mathrm{S}}^{*}\right)\right]_{s}$. Thus there exists $\Delta_{s}>0$ such that $\left[\mathbf{P}_{\mathrm{S}}^{*}\right]_{s}-\Delta_{s} \geq$ $\left[\mathbf{I}_{1}\left(\mathbf{P}_{\mathrm{S}}^{*}\right)\right]_{s}$. Moreover, decreasing $\left[\mathbf{P}_{\mathrm{S}}^{*}\right]_{s}$ by $\Delta_{s}$ create another feasible power allocation with less cost, which contradicts to the assumption. Suppose there exists $s$ such that $\left[\mathbf{P}_{\mathrm{S}}^{*}\right]_{s}>$ $\left[\mathbf{I}_{2}\left(\mathbf{P}_{\mathrm{S}}^{*}, \boldsymbol{\alpha}^{*}\right)\right]_{s}$ or $\left[\mathbf{P}_{\mathrm{S}}^{*}\right]_{s}>\left[\mathbf{I}_{3}\left(\mathbf{P}_{\mathrm{S}}^{*}, \boldsymbol{\alpha}^{*}\right)\right]_{s}$. Thus there exists $\boldsymbol{\alpha}^{\prime}$ such that $\left[\mathbf{I}_{2}\left(\mathbf{P}_{\mathrm{S}_{-s}}{ }_{-s}, \boldsymbol{\alpha}^{\prime}\right)\right]_{s}=\left[\mathbf{I}_{3}\left(\mathbf{P}_{\mathrm{S}_{-s}^{*}}^{*}, \boldsymbol{\alpha}^{\prime}\right)\right]_{s}<\left[\mathbf{P}_{\mathrm{S}}^{*}\right]_{s}$, which contradicts to the assumption. As a result, the optimal solution must fulfill the constraints at equality.

Now we show the optimal power allocation is unique. Suppose there exists other $\boldsymbol{\alpha}^{\prime}$ and $\mathbf{P}_{\mathrm{S}}^{\prime}$ such that $\mathbf{I}_{i}$ hold at equality for $i=\{1,2,3\}$. We can assume there exists $s$ such that $\left[\mathbf{P}_{\mathrm{S}}^{*}\right]_{s}<\left[\mathbf{P}_{\mathrm{S}}^{\prime}\right]_{s}$. Hence there exists $\delta>1$, such that $\delta \mathbf{P}_{\mathrm{S}}^{*} \geq \mathbf{P}_{\mathrm{S}}^{\prime}$ and $\delta\left[\mathbf{P}_{\mathrm{S}}\right]_{s}=\left[\mathbf{P}_{\mathrm{S}}^{\prime}\right]_{s}$. The monotonicity and scalability of standard interference function imply that

$$
\left[\mathbf{P}_{\mathrm{S}}^{\prime}\right]_{s}=\left[\mathbf{I}_{i}\left(\mathbf{P}_{\mathrm{S}}^{\prime}\right)\right]_{s} \leq\left[\mathbf{I}_{i}\left(\delta \mathbf{P}_{\mathrm{S}}^{*}\right)\right]_{s}<\delta\left[\mathbf{I}_{i}\left(\mathbf{P}_{\mathrm{S}}^{*}\right)\right]_{s}=\delta\left[\mathbf{P}_{\mathrm{S}}^{*}\right]_{s},
$$

which contradicts the assumption. It completes the proof.

Now we can prove Proposition 3.

Proof: Relax the constraints on capacity of cooperative primal users in problem (13) replacing $C_{p, s}^{\text {cop }}$ by $C_{p s 2 s^{\prime}}$. Then, rearrange the constraints by placing $P_{s}$ in the left side of the inequality only. Thus we get problem (14).

Suppose $\boldsymbol{\alpha}^{*}$ and $\mathbf{P}_{\mathrm{S}}^{*}$ are optimal solution for problem (14). Thus the constraints hold at equality according to Lemma 4. Moreover, if $\boldsymbol{\alpha}^{*}$ and $\mathbf{P}_{\mathrm{S}}^{*}$ are feasible for problem (13), they are also the optimal solution for it, which minimize the transmission power. Otherwise, suppose $\boldsymbol{\alpha}^{\prime}$ and $\mathbf{P}_{\mathrm{S}}^{\prime}$ are feasible and optimal solutions for problem (13). Follow similar proof in Lemma 4, we can show that constraints (14b), (14c) and (14d) hold at equality with $\boldsymbol{\alpha}^{\prime}$ and $\mathbf{P}_{\mathrm{S}}^{\prime}$, which contradicts the uniqueness given by Lemma 4 . It completes the proof.

Proposition 3 indicates that for a given $t$, which is feasible to problem (13), the optimal solutions for problem (13) can be determined by solving problem (14). Formally, we propose a set of equations that represents the equalities described by Lemma 4 for optimal solutions. Consider transmitter $i$ and receiver $j$. Normalize the antenna gain as $H_{c j}=G_{c j} / G_{i j}$ for either $c \in \mathcal{P}$ or $c \in \mathcal{S}$. Let $q_{i j}$ denote the normalized interference from transmitter $i \in \mathcal{S}$ to receiver $j$, then:

$$
q_{i j}=\eta_{i j}+I_{i j} / G_{i j},
$$

where $\eta_{j}=\sigma^{2} / G_{i j}$. In matrix notation, it can be written as

$$
\mathbf{q}=\mathbf{H}_{1} \tilde{\mathbf{Q}}_{\mathrm{P}}+\mathbf{H}_{2} \tilde{\mathbf{P}}_{S}+\boldsymbol{\eta},
$$

where $\mathbf{H}_{1}$ and $\mathbf{H}_{2}$ are defined respectively as:

$$
\left[\mathbf{H}_{1}\right]_{i j}=\left\{\begin{array}{ll}
0 & \text { if } \beta_{i}^{j}=1, j \in \mathcal{S}, i \in \mathcal{P} \\
0 & \text { if } i^{\prime}=j \\
G_{i j^{\prime}} / 2 G_{j j^{\prime}} & \text { if } \sum_{s} \beta_{i}^{s}=1, i \in \mathcal{P}, i \neq j^{\prime} \\
G_{i j^{\prime}} / G_{j j^{\prime}} & \text { otherwise }
\end{array},\right.
$$

and

$$
\left[\mathbf{H}_{2}\right]_{i j}=\left\{\begin{array}{ll}
0 & \text { if } \beta_{i}^{p_{i}}=1, i, j \in \mathcal{S} \\
0 & \text { if } i^{\prime}=j \\
G_{i j^{\prime}} / 2 G_{j j^{\prime}} & \text { if } \sum_{p} \beta_{p}^{i}=1, i \in \mathcal{S}, j \neq i^{\prime} \\
G_{i j^{\prime}} / G_{j j^{\prime}} & \text { otherwise }
\end{array} .\right.
$$

Note that vector $\mathbf{q} \in \mathbb{R}^{n+N_{S}}$ collects the normalized interference for the cases where transmitters are secondary users, and matrices $\mathbf{H}_{1} \in \mathbb{R}^{n+N_{S} \times N_{P}}, \mathbf{H}_{2} \in \mathbb{R}^{n+N_{S} \times n+N_{S}}$ collects normalized antenna gains constructed with respect to the primary and secondary users respectively. $n$ is the number of primary pairs that use secondary users as relays. Moreover, $\tilde{\mathbf{P}}_{S}$ represents the power allocation for all secondary users, which is formally defined as

$$
\tilde{\mathbf{P}}_{S}=\left(\tilde{\mathbf{P}}_{S}^{\alpha} ; \tilde{\mathbf{P}}_{S}^{1-\alpha}\right),
$$

where $\tilde{\mathbf{P}}_{S}^{\alpha}$ collects the power allocations for helping primary users, while $\tilde{\mathbf{P}}_{S}^{1-\alpha}$ collects the those for transmission for secondary pairs.

For example, suppose there are 2 primary users, $p_{1}, p_{2}$, and 2 secondary users, $s_{1}$ and $s_{2}$. User $s_{2}$ cooperates with primary user $p_{1}$. Thus

$$
\begin{aligned}
& \mathbf{H}_{1}=\left[\begin{array}{cc}
0 & G_{p_{2} p_{1}^{\prime}} / G_{s_{2} p_{1}^{\prime}} \\
G_{p_{1} s_{1}^{\prime}} / 2 G_{s_{1} s_{1}^{\prime}} & G_{p_{2} s_{1}^{\prime}} / G_{s_{1} s_{1}^{\prime}} \\
0 & G_{p_{2} s_{2}^{\prime}} / G_{s_{2} s_{2}^{\prime}}
\end{array}\right], \\
& \mathbf{H}_{2}=\left[\begin{array}{ccc}
0 & G_{s_{1} p_{1}^{\prime}} / G_{s_{2} p_{1}^{\prime}} & 1 \\
G_{s_{2} s_{1}^{\prime}} / 2 G_{s_{1} s_{1}^{\prime}} & 0 & G_{s_{2} s_{1}^{\prime}} / 2 G_{s_{1} s_{1}^{\prime}} \\
1 & G_{s_{1} s_{2}^{\prime}} / G_{s_{2} s_{2}^{\prime}} & 0
\end{array}\right],
\end{aligned}
$$

while $\tilde{\mathbf{P}}_{S}=\left(\alpha_{s_{2}} P_{s_{2}}, P_{s_{1}},\left(1-\alpha_{S_{2}}\right) P_{s_{2}}\right)^{T}$. Note that in this case if we have the optimal solution for $\tilde{\mathbf{P}}_{S}$, then the optimal $\mathbf{P}_{S}$ is obtained.

We assume that $\mathbf{H}_{1}$ and $\mathbf{H}_{2}$ are primitive matrix in the rest of this work. Let $\gamma_{i j}$ be the SIR targeted by transmitter $i$ to receiver $j$. In this work, the target SIR could be semidetermined as:

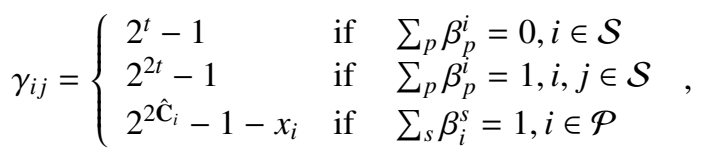

where $x_{i}$ is defined as

$$
x_{i}=\frac{Q_{p} G_{i i^{\prime}}}{\sigma^{2}+I_{i i^{\prime}}},
$$


which is so far unknown and can be obtained iteratively by Newton's method. With the above notation, $\gamma_{t}=P_{t} / q_{r}$, or equivalently,

$$
\tilde{\mathbf{P}}_{S}=\mathbf{D}(\gamma) \mathbf{q},
$$

where $\mathbf{D}(\gamma)=\operatorname{diag}\left(\gamma_{1}, \ldots, \gamma_{N_{r}}\right)$. Combining Eq. (15) and (16), we get the following equations:

$$
\begin{aligned}
\mathbf{q} & =\mathbf{H}_{1} \tilde{\mathbf{Q}}_{\mathbf{P}}+\mathbf{H}_{2} \mathbf{D}(\gamma) \mathbf{q}+\boldsymbol{\eta} \\
\tilde{\mathbf{P}}_{S} & =\mathbf{D}(\gamma) \mathbf{H}_{1} \tilde{\mathbf{Q}}_{\mathbf{P}}+\mathbf{D}(\gamma) \mathbf{H}_{2} \tilde{\mathbf{P}}_{S}+\mathbf{D}(\gamma) \boldsymbol{\eta} .
\end{aligned}
$$

Recall the standard result [11]:

Proposition 5. Suppose $\mathbf{H}_{1} \tilde{\mathbf{Q}}_{P}+\boldsymbol{\eta}>0$. A SIR vector $\boldsymbol{\gamma}>0$ is feasible if and only if $\rho\left(\mathbf{H}_{2} \mathbf{D}(\gamma)\right)<1$

Denote $\tilde{\mathbf{P}}_{S}(\boldsymbol{\gamma})$ the power allocation corresponding to $\boldsymbol{\gamma}$, and it is determined by

$$
\tilde{\mathbf{P}}_{S}(\boldsymbol{\gamma})=\left(I-\mathbf{D}(\boldsymbol{\gamma}) \mathbf{H}_{2}\right)^{-1} \mathbf{D}(\gamma)\left(\mathbf{H}_{1} \tilde{\mathbf{Q}}_{\mathrm{P}}+\boldsymbol{\eta}\right) .
$$

Based on this observation, it is easy for the central controller to determine whether $t$ is feasible for problem (12), and further obtain the power allocations for secondary users, which summarized in Algorithm 1 that uses $\boldsymbol{\beta}$ as input and provides $\mathbf{P}_{S}, \boldsymbol{\alpha}$ and $t$ as outputs. Note that $\tilde{\mathbf{P}}_{S}$ is feasible only if all its entries are positive and $P_{s} \leq P_{s}^{\max }$ for all secondary users, and $C_{p} \geq \hat{C}_{p}$ for all primary users.

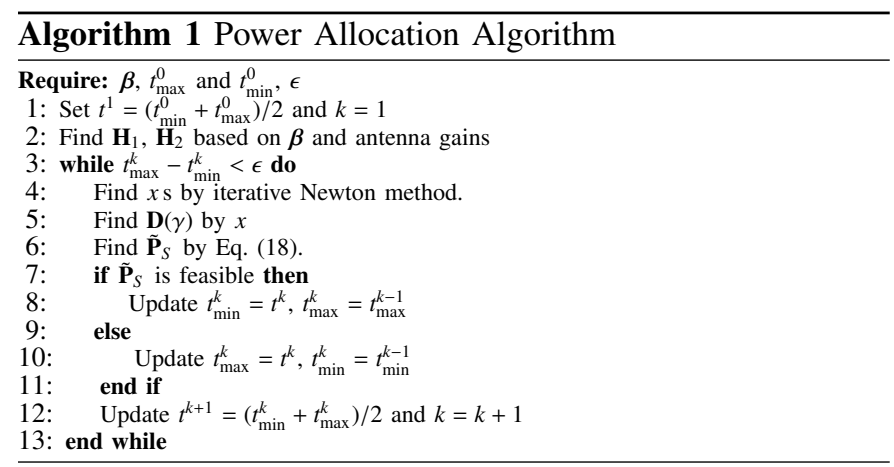

\section{B. Secondary User Selection Problem}

In previous section, we introduce the method to find the optimal solutions $\mathbf{P}_{\mathrm{S}}^{*}$ and $\boldsymbol{\alpha}^{*}$ and $t^{*}$ when $\boldsymbol{\beta}$ is fixed. In this section, we introduce the method that finds the optimal secondary selection for the primal user. However, this secondary selection problem is mixed integer programming problem. Generally it is NP hard problem. In this section, we will introduce a greedy algorithm that provides the sub-optimal solution for the secondary selection based on local search.

From Lemma 1, after running Algorithm 1 there exists secondary user $s$ such that $P_{s}=P_{s}^{\max }$ or primal user $p$ such that $C_{\mathrm{p}}\left(\mathbf{P}_{\mathrm{S}}^{*}, \boldsymbol{\alpha}^{*}\right)=\hat{C}_{\mathrm{p}}$. Thus, secondary user $s$ or primary user $p$ is the bottle-neck of the network. Furthermore, search possible cooperation for available secondary users. To summarize, we let the central controller search possible better $\boldsymbol{\beta}$ associated with $s$ or $p$, such that the new solutions $\tilde{\mathbf{P}}_{S}$ to Eq. (17) are strictly less than $P^{\max }$, and $C_{\mathrm{p}}\left(\mathbf{P}_{\mathrm{S}}^{*}, \boldsymbol{\alpha}^{*}\right)=\hat{C}_{\mathrm{p}}$ for all $p$. If there is no better associations, central control searches better $\boldsymbol{\beta}$ for a randomly pick secondary user that is not cooperated with any primary users.

Note that the central controller could solve problem (17) efficiently and check the feasibility of $t$ easily as described by Algorithm 1. It is possible for the central controller to find the optimal secondary selection by brute-force search. However, the combination of all possible selections is quiet large and depends on the size of the network. In this case, we enforce the central controller only find a better secondary selection at each time. It needs only search a smaller feasible solution in each iteration. Thus the algorithm is designed to find a continuous improved path. Algorithm 2 summarize the strategy, in which $\delta$-improvement ensures that when $\beta$ updates, $t$ increases at least $\delta$.

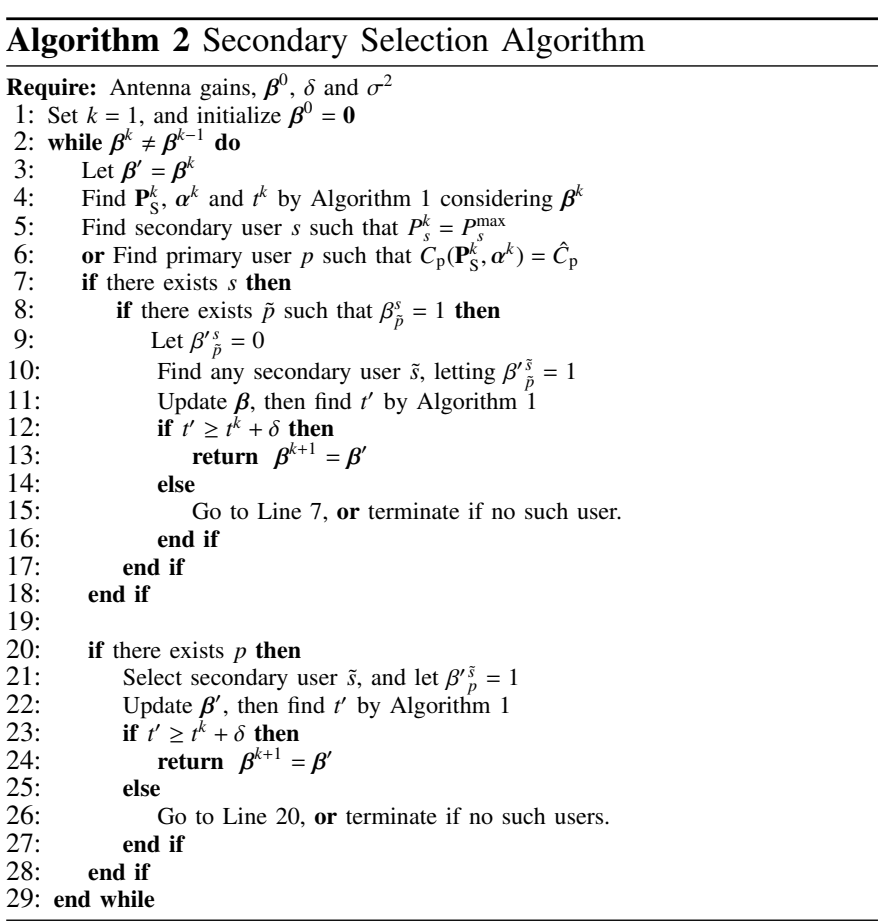

Now we have following proposition:

Proposition 6. The secondary user selection, described by Algorithm 2, has finite improvement, and terminate in finite time.

Proof: From the result from Lemma 4, we have that if there doesn't exist $P_{s}=P_{s}^{\max }$ and $C_{\mathrm{p}}\left(\mathbf{P}_{\mathrm{S}}^{*}, \boldsymbol{\alpha}^{*}\right)=\hat{C}_{\mathrm{p}}$, then there exists some $\Delta t>0$, such that $t+\Delta t$ is feasible to problem (14). It indicates that any change in the $\boldsymbol{\beta}$ improves the system performance. Furthermore, note that the improvement is at least larger than threshold $\delta$. Since the optimal utility value is finite, thus the number of improvement must be finite as well. Thus, the strategy will terminate in finite time.

\section{Numerical Results}

Consider a communication area of size $L^{2}$, which is set to $1500 \times 1500 \mathrm{~m}^{2}$, in which primary and secondary users are placed randomly as illustrated in Fig. 3. In the simulations, the antenna gain between transmitter $i$ and receiver $j$, is 


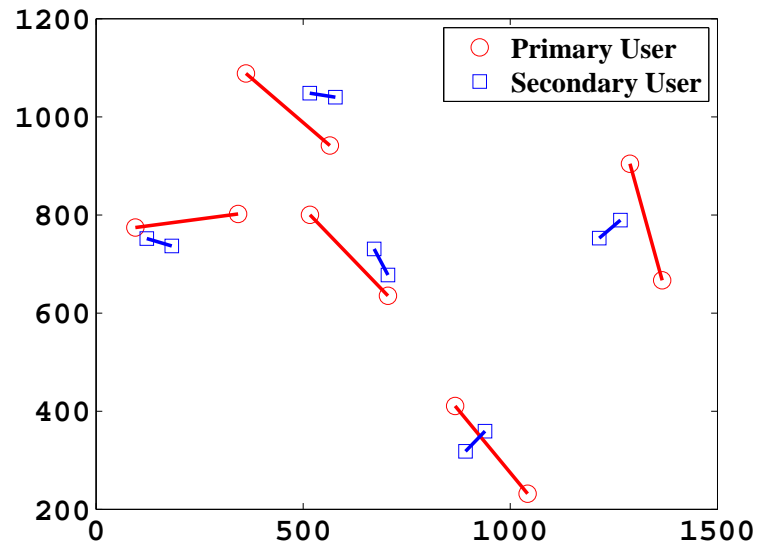

Fig. 3. An example of the topologies used in simulations.

TABLE I

SimULATION PARAMETERS

\begin{tabular}{|c|l|c|}
\hline Parameter & Description & Value (unit) \\
\hline$L^{2}$ & Communication Area & $1500 \times 1500 \mathrm{~m}^{2}$ \\
\hline$m$ & Path loss factor & 4 \\
\hline$P_{s}^{\max }$ & Maximum power for secondaries & $0.5 \mathrm{~W}$ \\
\hline$\tilde{Q}_{p}$ & Transmission power for primaries & $1 \mathrm{~W}$ \\
\hline$\sigma^{2}$ & Noise power & $10^{-10} \mathrm{~W}$ \\
\hline$B$ & Bandwidth & $64 \mathrm{kHz}$ \\
\hline$N_{P}$ & Number of primary users & 10 \\
\hline$N_{S}$ & Number of secondary users & 10 \\
\hline
\end{tabular}

approximately determined by the distance between $i$ and $j$, i.e. ,

$$
G_{i j}=d(i, j)^{-m},
$$

where $m$ is the path loss factor, normally $m \in[2,6]$, and $d(i, j)$ is the distance between transmitter $i$ and receiver $j$. The maximum transmission power for each secondary user, $P_{s}^{\max }$, is set to $0.5 \mathrm{~W}$, while the power for each primary user is set to $1 \mathrm{~W}$. The average noise power, $\sigma^{2}$, is assumed to be $10^{-10} \mathrm{~W}$. Rather than the unit bandwidth used in problem formulation, the bandwidth, $B$, of each link is set to $64 \mathrm{kHz}$. The margin gain $\rho$ is varying for comparison. The parameters are summarized in Table I. Note that the simulation parameters used in this paper are chosen similarly to those used in [12], in which a similar cognitive network was considered.

The performance of the proposed algorithm is evaluated by comparing to the performance achieved by the strategy in which cooperation between primary and secondary users is not allowed. Different primary capacity requirements margin gains are considered, and numerical results are obtained by averaging over 30 independent simulations for different network topologies. Fig. 4 illustrates the numerical results. The proposed algorithm benefits around from 2 to 5 times better than the non-cooperation strategy for secondary users.

\section{Conclusion}

Guaranteeing the performance of primary users, a maximum minimum optimization problem is proposed for secondary users. Fixing the association between primary and secondary users, an optimal algorithm is introduced to find the largest

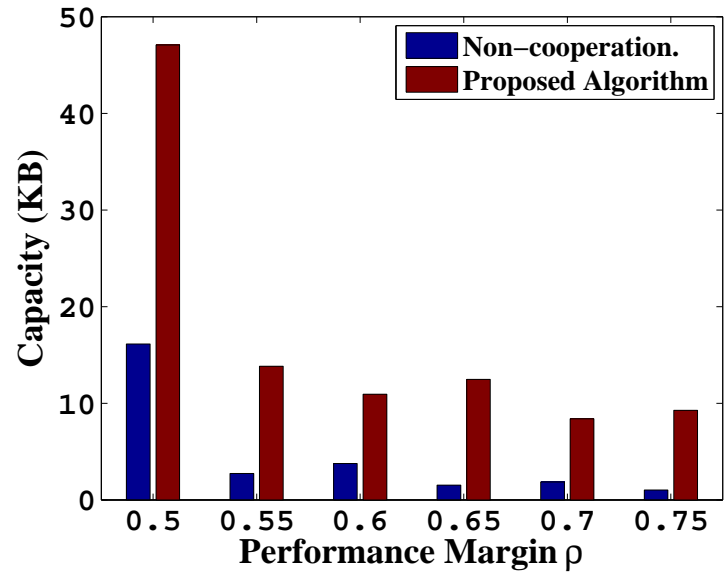

Fig. 4. Performance comparison between the proposed algorithm and the strategy that does not allow cooperation. Note that we use different $\hat{C}_{\mathrm{P}}$ 's in different cases.

feasible link capacity for secondary users. A greedy algorithm is proposed in a central controller to find a sub-optimal secondary user selections for primary users.

In future work, we will try to find the distributed algorithms to determine the largest feasible capacity for secondary users, and to find optimal secondary selections for primary users.

\section{REFERENCES}

[1] O. Simeone, I. Stanojev, S. Savazzsi, Y. Bar-Ness, U. Spagnoloni, and R. Pickholtz, "Spectrum leasing to cooperative secondary ad hoc networks," IEEE J. Sel. Areas Commun, vol. 26, no. 1, pp. 203-213, Jan. 2008.

[2] J. Zhang and Q. Zhang, "Stackelberg game for utility-based cooperative cognitive radio networks," in Proc. ACM MobiHoc 2009.

[3] D. Li, X. Wang, and M. Guizani, "Coalitional game theoretic approach for secondary spectrum access in cooperative cgnitive radio networks," IEEE Trans. Wireless Commun., vol. 10, no. 3, pp. 844-856, Mar. 2011.

[4] K. Khalil, M. Karaca, O. Ercetin, and E. Ekici, "Optimal scheduling in cooperate-to-join cognitive radio networks," in Proc. IEEE INFOCOM 2011.

[5] Y. Han, A. Pandharipande, and S. H. Ting, "Cooperation decode-andforward relaying for secondary spectrum access," IEEE Trans. Wireless Commun., vol. 8, no. 10, pp. 4945-4950, Oct. 2009.

[6] Y. Han, S. H. Ting, and A. Pandharipande, "Cooperative spectrum sharing protocol with secondary user selection," IEEE Trans. Wireless Commun., vol. 9, no. 9, pp. 2914-2923, Sep. 2010.

[7] L. Ding, T. Melodia, S. N. Batalama, and J. D. Matyjas, "Distributed routing, relay selection, and spectrum allocation in cognitive and cooperative ad hoc networks," in Proc. IEEE Secon 2011.

[8] Z. Guan, T. melodia, D. Yuan, and D. A. Pados, "Distributed spectrum management and relay selection in interference-limited cooperative wireless networks," in Proc. IEEE MobiHoc 2011.

[9] B. Maham, P. Popovski, X. yun Zhou, and A. Hjorungnes, "Cognitive multiple access network with outage margin in the primary system,"

[10] R. Yates, "A framework for uplink power control in cellular radio systems," Selected Areas in Communications, IEEE Journal on, vol. 13, no. 7, pp. 1341-1347, 1995.

[11] J. Zander, "Performance of optimum transmitter power control in cellular radio systems," Vehicular Technology, IEEE Transactions on, vol. 41, pp. 57-62, Feb 1992.

[12] Z. Guan, T. Melodia, D. Yuan, and D. A. Pados, "Distributed spectrum management and relay selection in interference-limited cooperative wireless networks," in Proceedings of the 17th Annual International Conference on Mobile Computing and Networking, (New York, NY, USA), pp. 229-240, ACM, 2011. 